\title{
Non-intubated uniportal video-assisted thoracoscopic surgery: lobectomy and systemic lymph node dissection
}

\author{
Haoyou Wang, Jijia Li, Yu Liu, Gebang Wang, Pingwen Yu, Hongxu Liu \\ Department of Thoracic Surgery, Cancer Hospital of China Medical University, Liaoning Cancer Hospital \& Institute, Shenyang, China \\ Correspondence to: Hongxu Liu. Department of Thoracic Surgery, Cancer Hospital of China Medical University, Liaoning Cancer Hospital \& \\ Institute, Shenyang 110042, China. Email: hxliu@cmu.edu.cn.
}

Submitted Apr 22, 2020. Accepted for publication Aug 03, 2020.

doi: $10.21037 /$ jtd-20-1703

View this article at: http://dx.doi.org/10.21037/jtd-20-1703

\section{Introduction}

In the past decade, there has been a resurgence of nonintubated thoracic surgery (NITS), given its enhanced recovery after surgery (ERAS). Airway injury and other complications from tracheal intubation and one-lung ventilation (OLV) are often serious (1). The main advantage of NITS is that it avoids airway damage caused by OLV and other perioperative complications $(2,3)$. NITS reportedly can be applied to most video-assisted thoracoscopic surgery (VATS) operations, including uniportal thoracic surgery (4). The development of uniportal thoracoscopy technology in recent years has further reduced patient trauma, shortened the length of stay, and reduced hospital costs $(5,6)$. The combination of uniportal VATS and non-intubated anesthesia further augments these advantages and enables full implementation of rapid recovery (7). Here, we present a video of non-intubated uniportal VATS comprising left lower lobectomy and systemic lymph node dissection.

\section{Surgical technique}

The patient was a 37-year-old woman with a $4 \mathrm{~cm}$ tumor located on the left lower lobe (LLL). Enhanced thin-layer CT showed visceral pleural indentation with no obvious enlarged hilar and mediastinal lymph nodes, clinical TNM staging cT2N0M0. After multi-disciplinary discussions, the patient's indications for surgery were clear and there were no contraindications to NITS surgery. The video shows a left lower lobectomy and systemic lymph node dissection. The patient was administered midazolam and atropine, 30 minutes before the start of anesthesia. The patient's heart rate, electrocardiogram waveform, respiratory rate, pulse oximetry, and blood pressure were checked through the anesthesia monitor. Anesthesia in NITS includes local anesthesia and selective intercostal nerve block (8). The surgeon stood on the patient's abdominal side (i.e., in front of the patient) for surgical operations, while the assistant stood on the patient's backside (i.e., behind the patient). First, the anterior axillary surgical incision was infiltrated with $0.5 \%$ ropivacaine at the level of the fifth intercostal space. Subsequently, a $4 \mathrm{~cm}$ incision was made. The iatrogenic pneumothorax caused by a surgical incision helped the lung tissue to collapse on one side of the operation. To achieve adequate anesthesia, $0.5 \%$ ropivacaine was administered between third and eighth intercostal spaces. The irritating cough produced during the dissection of blood vessels under NITS is an important risk to patient safety. Production of this irritating cough can be reduced by spraying lidocaine or ropivacaine on the surface of the pleura (9). In this surgery, $0.5 \%$ ropivacaine was sprayed on the pleural surface; $2 \%$ lidocaine was used for vagus nerve block under direct thoracoscopic vision to effectively abolish the cough reflex. After anesthesia had been induced, the surgical procedures of left lower anatomical lobectomy and systemic lymph node dissection were initiated; the lymph nodes involved in this operation were $4 \mathrm{L \#}, 5 \#, 6 \#$, 7\#, 8\#, 9\#, 10\#, 11\#, 12\#, 13\# and 14\# (Video 1). The patient was permitted to drink or consume liquid food 6 hours after the operation.

\section{Discussion}

Contraindications and indications for non-intubated uniportal VATS are related to the past experiences of 
each medical team and the characteristics of each patient. Absolute contraindications are that the medical team is incapable of performing the surgery and the patient refuses treatment. Medical team-related relative contraindications include technical contraindications to general anesthesia, difficult airway management, requirement for protection of the contralateral lung from spillage of endobronchial contents, and inexperienced or uncooperative team members. Patient-related relative contraindications include obesity, allergy to local anesthetic, neurological conditions, uncontrolled gastroesophageal regurgitation or elevated risk of regurgitation ( $<6$ hours fasting), central hypoventilation syndrome, persistent cough or high airway secretion, hemodynamic instable or severe hypoxia/hypercapnia, high risk of adhesions and history of surgery or radiation therapy $(6,10)$. If the patient is undergoing NITS, the patient's body mass index (BMI) must be $\leq 25$, and the American Society of Anesthesiologists (ASA) status should be grade I or II.

Compared with the traditional VATS method, patients undergoing non-intubated uniportal VATS reportedly have shorter postoperative hospital stays, earlier postoperative meals, and earlier resumption of daily activities (11). The choice of a single-incision technique, combined with the NITS, further reduces damage to patients (12). We used a laryngeal mask during surgery to facilitate safer respiratory management. Ultimately, the goal of surgery is improvement of patient prognosis, and which allows patients to be discharged safely and quickly, thereby fully implementing ERAS. We have demonstrated that this nonintubation method is feasible for lobectomy and systemic lymph node dissection by using strict enrollment criteria and careful methods; our postoperative results have been good and postoperative hospital stay has been shorter.

An emergency intubation treatment plan must be developed before NITS to ensure patient safety. An intraoperative switch to general anesthesia with intubation is unavoidable in some instances; the treatment team must therefore develop alternative plans to minimize the patient's risk. General anesthesia with intubation is recommended for patients with severe adhesions, considerable mediastinal movements, major bleeding, persistent hypoxemia, or respiratory distress during surgery. To reduce the rate of intraoperative conversion to intratracheal intubation, we recommend strict implementation of the admission criteria described above. Notably, some surgical teams and anesthesiologists cannot perform non-intubated uniportal VATS. It is important to ensure that non-intubated pulmonary resections and systemic lymph node dissection are only be performed by experienced thoracoscopic surgeons and anesthetists.

\section{Acknowledgments}

Funding: This work was supported by the construction of Liaoning Cancer Research Center (Lung Cancer) (2019JH6/10200011), a technological particular project of Liaoning Province of China (2019020176-JH1/103), the central financial fund for promoting medical service and safeguarding capability (capability construction of medical and health organizations), a subsidy to the construction of Provincial Key Specialty, a research grant to introduced talents of Liaoning Cancer Hospital, Liaoning Province Doctor Startup Fund (2019-BS-148).

\section{Footnote}

Conflicts of Interest: All authors have completed the ICMJE uniform disclosure form (available at http://dx.doi. org/10.21037/jtd-20-1703). The authors have no conflicts of interest to declare.

Ethical Statement: The authors are accountable for all aspects of the work in ensuring that questions related to the accuracy or integrity of any part of the work are appropriately investigated and resolved. The study conformed to the provisions of the Declaration of Helsinki (as revised in 2013), written informed consent for participation was obtained from each participant.

Open Access Statement: This is an Open Access article distributed in accordance with the Creative Commons Attribution-NonCommercial-NoDerivs 4.0 International License (CC BY-NC-ND 4.0), which permits the noncommercial replication and distribution of the article with the strict proviso that no changes or edits are made and the original work is properly cited (including links to both the formal publication through the relevant DOI and the license). See: https://creativecommons.org/licenses/by-nc-nd/4.0/.

\section{References}

1. Murphy GS, Szokol JW, Avram MJ, et al. Postoperative residual neuromuscular blockade is associated with impaired clinical recovery. Anesth Analg 2013;117:133-41.

2. He J, Liu J, Zhu C, et al. Expert consensus on tubeless video-assisted thoracoscopic surgery (Guangzhou). J 
Thorac Dis 2019;11:4101-8.

3. He J, Liu J, Zhu C, et al. Expert consensus on spontaneous ventilation video-assisted thoracoscopic surgery in primary spontaneous pneumothorax (Guangzhou). Ann Transl Med 2019;7:518.

4. Zhao ZR, Lau RWH, Ng CSH. Anaesthesiology for uniportal VATS: double lumen, single lumen and tubeless. J Vis Surg 2017;3:108.

5. Bedetti B, Patrini D, Bertolaccini L, et al. Uniportal nonintubated thoracic surgery. J Vis Surg 2018;4:18.

6. Rocco G. Non-intubated uniportal lung surgery. Eur J Cardiothorac Surg 2016;49 Suppl 1:i3-5.

7. Mineo TC, Sellitri F, Fabbi E, et al. Uniportal nonintubated lung metastasectomy. J Vis Surg 2017;3:118.

8. Sihoe AD, Manlulu AV, Lee TW, et al. Pre-emptive local anesthesia for needlescopic video-assisted thoracic surgery: a randomized controlled trial. Eur J Cardiothorac Surg 2007;31:103-8.

9. Kiss G, Castillo M. Nonintubated anesthesia in thoracic surgery: general issues. Ann Transl Med 2015;3:110.

10. Irons JF, Martinez G. Anaesthetic considerations for nonintubated thoracic surgery. J Vis Surg 2016;2:61.

11. Liu J, Cui F, Li S, et al. Nonintubated video-assisted thoracoscopic surgery under epidural anesthesia compared with conventional anesthetic option: a randomized control study. Surg Innov 2015;22:123-30.

12. Gonzalez-Rivas D, Aymerich H, Bonome C, et al. From Open Operations to Nonintubated Uniportal Video-Assisted Thoracoscopic Lobectomy: Minimizing the Trauma to the Patient. Ann Thorac Surg 2015;100:2003-5.
Cite this article as: Wang H, Li J, Liu Y, Wang G, Yu P, Liu H. Non-intubated uniportal video-assisted thoracoscopic surgery: lobectomy and systemic lymph node dissection. J Thorac Dis 2020;12(10):6039-6041. doi: 10.21037/jtd-20-1703 